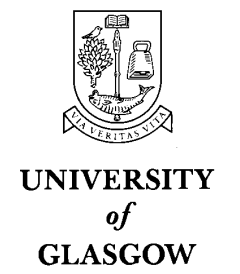

Department of Physics \& Astronomy Experimental Particle Physics Group

Kelvin Building, University of Glasgow,

Glasgow, G12 8QQ, Scotland

Telephone: +44 (0)1413398855 Fax: +44 (0)141 3305881
GLAS-PPE/2006-01

$24^{\text {th }}$ January 2006

\title{
Isolated leptons with missing transverse momentum and search for single top production
}

\author{
James Ferrando \\ ${ }^{1}$ University of Glasgow, Glasgow, G12 8QQ, Scotland
}

\begin{abstract}
Recent results of searches for production of isolated leptons in events with large missing transverse momentum in $e^{ \pm} p$ collisions at HERA are reviewed. The H1 collaboration observes an excess of these events over the, W-production dominated, Standard Model (SM) predictions in $e^{+} p$ collisions, this excess is not confirmed by ZEUS. In $e^{-} p$ collisions the rate of these events observed by the H1 collaboration is consistent with the Standard Model predictions. A potential new-physics source of isolated leptons in events with large missing transverse momentum at HERA is single top production via flavour changing neutral currents (FCNC). The most recent results of searches for this process from the H1 collaboration are presented including limits on the FCNC couplings. The HERA limits remain the strongest on photonic FCNC.
\end{abstract}


Table 1: The numbers of observed events in different $P_{T}^{X}$ ranges in the $\mathrm{H} 1$ isolated high $p_{T}$ lepton search compared to the SM expectations. The fraction of single- $W$ production included in the expectation is indicated in parentheses. The statistical and systematic uncertainties, added in quadrature, are also indicated.

\begin{tabular}{|c|c|c|c|c|}
\hline \multicolumn{2}{|c|}{ H1 Preliminary } & $\begin{array}{c}\text { Electron } \\
\text { obs./exp. }(W)\end{array}$ & $\begin{array}{c}\text { Muon } \\
\text { obs./exp. }(W)\end{array}$ & $\begin{array}{c}\text { Combined } \\
\text { obs./exp. }(W)\end{array}$ \\
\hline \hline $1994-2004$ & Full Sample & $19 / 14.6 \pm 2.0(70 \%)$ & $9 / 3.9 \pm 0.6(84 \%)$ & $28 / 18.5 \pm 2.7(73 \%)$ \\
\cline { 2 - 5 } $158 \mathrm{pb}^{-1} e^{+} p$ & $p_{T}^{X}>25 \mathrm{GeV}$ & $9 / 2.3 \pm 0.4(80 \%)$ & $6 / 2.3 \pm 0.4(84 \%)$ & $15 / 4.6 \pm 0.8(82 \%)$ \\
\hline \hline $1998-2005$ & Full Sample & $6 / 5.8 \pm 0.9(62 \%)$ & $0 / 1.5 \pm 0.5(76 \%)$ & $6 / 7.3 \pm 1.4(65 \%)$ \\
\cline { 2 - 5 } $53 \mathrm{pb}^{-1} e^{-} p$ & $p_{T}^{X}>25 \mathrm{GeV}$ & $2 / 0.9 \pm 0.2(71 \%)$ & $0 / 0.9 \pm 0.2(73 \%)$ & $2 / 1.8 \pm 0.3(72 \%)$ \\
\hline \hline $1994-2005$ & Full Sample & $25 / 20.4 \pm 2.9(68 \%)$ & $9 / 5.4 \pm 1.1(82 \%)$ & $34 / 25.7 \pm 4.0(71 \%)$ \\
\cline { 2 - 5 } $211 \mathrm{pb}^{-1} e^{ \pm} p$ & $p_{T}^{X}>25 \mathrm{GeV}$ & $11 / 3.2 \pm 0.6(77 \%)$ & $6 / 3.2 \pm 0.5(81 \%)$ & $17 / 6.4 \pm 1.1(79 \%)$ \\
\hline
\end{tabular}

Table 2: The numbers of observed events in the ZEUS isolated lepton search, compared to the SM expectations. The fraction of single- $W$ production included in the expectation is indicated in parentheses. The statistical and systematic uncertainties added in quadrature are also indicated.

\begin{tabular}{|c|c|c|}
\hline Isolated $e$ candidates & $12<P_{T}^{X}<25 \mathrm{GeV}$ & $P_{T}^{X}>25 \mathrm{GeV}$ \\
\hline ZEUS (prel.) 99-00 $\left(66 \mathrm{pb}^{-1}\right)$ & $1 / 1.04 \pm 0.11(57 \%)$ & $1 / 0.92 \pm 0.09(79 \%)$ \\
\hline ZEUS (prel.) 03-04 $\left(40 \mathrm{pb}^{-1}\right)$ & $0 / 0.46 \pm 0.10(64 \%)$ & $0 / 0.58_{-0.09}^{+0.08}(76 \%)$ \\
\hline
\end{tabular}

\section{Isolated leptons in events with missing transverse momentum}

The observation of anomalous events with an isolated high energy lepton and missing transverse momentum in ep collisions at HERA was reported by the H1 collaboration at the end of the 1994-2000 running period [1]. Production of single $W$ bosons with subsequent leptonic decay is the only process with a measurable cross section at HERA which leads to this type of events. The dominant leading order Feynman diagram for W production is shown in Fig.1(a); the total cross section is about $1 \mathrm{pb}$, evaluated including $\mathscr{O}\left(\alpha^{2} \alpha_{s}\right)$ QCD corrections [2].

New results in searches for isolated electrons and muons from H1 for the 1994-2005 running period are summarised in Table 1 [3]. An excess of observed data over the SM expectations can be seen in the H1 results, this is illustrated in Fig.1(b). This excess is actually observed only in the $e^{+} p$ data (see Table 1), it is not confirmed in a similar search by the ZEUS collaboration [4], which is summarised in Table 2. The excess over the SM prediction at high values of hadronic $P_{T}$ is suggestive of a heavy particle decay process. More specialised searches have been applied to look for high $p_{T}$ leptons arising from the leptonic decay channel of single-top production, as discussed in section 2.

A slight excess in the $\tau$ channel was reported by ZEUS for $130 \mathrm{pb}^{-1}$ of data from the 1994-2000 running period [5]. The results of the latest $\mathrm{H} 1$ search for isolated $\tau$ leptons using $108 \mathrm{pb}^{-1}$ of data from the 1996-2000 running period [6] are given in Table 3. No excess over the SM is observed.

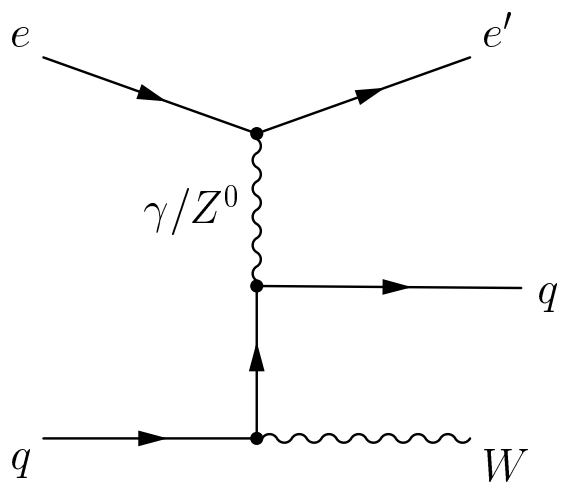

(a)

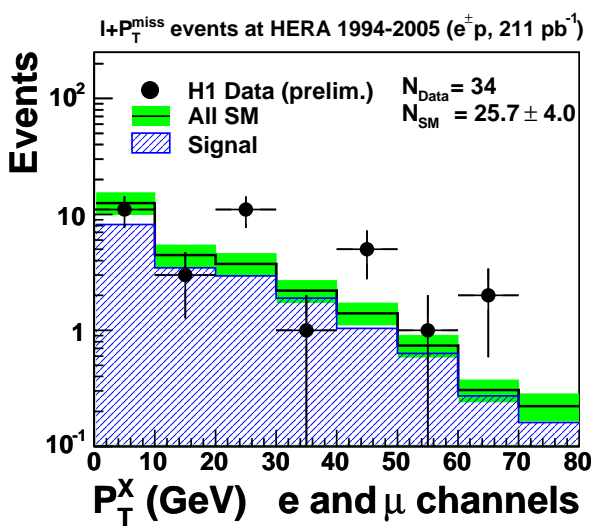

(b)

Figure 1: (a) LO Feynman diagram for single-W production at HERA. (b) Hadronic $P_{T}$ distribution of H1 isolated lepton and muon candidates. 
Table 3: The numbers of observed events in the $\mathrm{H} 1$ isolated $\tau$-lepton search, compared to the SM expectations. The fraction of single- $W$ production included in the expectation is indicated in parentheses. The statistical and systematic uncertainties added in quadrature are also indicated.

\section{Single-top production at HERA}

\begin{tabular}{|c|c|}
\hline $\begin{array}{c}\text { H1 Preliminary } \\
\text { 96-00 } 108 \mathrm{pb}^{-1}\end{array}$ & $\begin{array}{c}\text { Tau Channel } \\
\text { Obs./Exp.(W) }\end{array}$ \\
\hline \hline Full Sample & $5 / 5.81 \pm 1.36(15 \%)$ \\
$P_{T}^{X}>25 \mathrm{GeV}$ & $0 / 0.53 \pm 0.10(49 \%)$ \\
$P_{T}^{X}>40 \mathrm{GeV}$ & $0 / 0.22 \pm 0.05(54 \%)$ \\
\hline
\end{tabular}

In the SM, FCNC interactions are suppressed by the GIM mechanism [7]. Due to the large mass of the top quark, close to the electroweak symmetry breaking scale, deviations from the SM might be observed first in the top sector. At HERA, with its maximum centre-of-mass energy of $\sqrt{s}=318 \mathrm{GeV}$, top quarks can only be singly produced. At tree level, production proceeds through the charged current reaction $e p \rightarrow v t \bar{b} X$. Since the SM cross section for single-top production is less than $1 \mathrm{fb}$, any observed single-top events in the present data would be a clear sign of physics beyond the SM. The FCNC coupling, $t u V^{1)}$ or $t c V$, would induce the neutral current reaction $e p \rightarrow e t X[8,9]$, in which the incoming lepton exchanges a $\gamma$ or $Z^{0}$ with a $u$ quark in the proton, yielding a $t$ quark in the final state (Fig. 2(a)). The $Z^{0}$ exchange is suppressed by the large propagator mass. Furthermore, large values of Bjorken $x \gtrsim 0.3$ are needed to produce a top quark. Since the $u$-quark density of the proton is much higher than the $c$-quark density at large $x$, production of single top-quarks at HERA is most sensitive to a coupling of the type $t u \gamma$.

The SM decay, $t \rightarrow b W$, with subsequent leptonic decay of the $W$ boson, $W \rightarrow e v_{e}, \mu v_{\mu}$, leads to the presence of an isolated high-energy lepton, significant missing transverse momentum arising from the undetected neutrino and a large value of the hadronic transverse momentum, $p_{T}^{X}$, stemming from the $b$-quark decay. In the hadronic decay channel of the $W$ boson, $W \rightarrow q \bar{q}^{\prime}$ three jets are expected in the final state, with the dijet invariant-mass distribution for the correct pair of jets peaking at the $W$ mass, $M_{W}$, and the three-jet invariant-mass distribution peaking at the top quark mass, $M_{\mathrm{top}}$. QCD multijet events are the main SM background in the hadronic channel.

A search for single-top quark production at HERA was previously performed by the ZEUS collaboration [10] using the full HERA I dataset; no evidence for single top production was observed. The latest H1 Search [11] requires large missing total transverse momentum, the presence of a well isolated high- $p_{T}$ lepton and a high- $E_{T}$ jet, in the leptonic channel. In the hadronic channel 3 high- $E_{T}$ jets are required. In the leptonic channel 5 events are observed while $1.31 \pm 0.22$ are expected from the SM, whilst no excess over the SM is observed in the, less sensitive, hadronic channel. In both cases the kinematics of the $\mathrm{W}$ and $b$ are reconstructed and used to create a multivariate discriminator variable.

A limit on the cross section for top production is obtained from a combination of likelihood fits using the discriminator functions for the respective channels, and this is translated into a 95\% confidence limit on the anomalous $t u \gamma$ coupling. Fig. 2(b) shows the H1 limits, for LO signal calculation, compared to those set by the CDF [12], L3 [13] and ZEUS [10] experiments.

\section{Summary}

The H1 collaboration continues to observe anomalous events with an isolated high energy muon or electron and missing transverse momentum in $e^{+} p$ collisions at HERA. The probability for the SM expectation to fluctuate to the observed number of events or more in the high $P_{T}^{X}$ domain is 0.0012 compared to 0.0015 for the previously published HERA I data. ZEUS does not to confirm any excess over the SM in the production of these events. The H1 collaboration observe no excess in the $\tau$ channel. A search for a possible new physics source of these events, single top production, by $\mathrm{H} 1$ has been used to set upper limits on the FCNC coupling $t u \gamma$ to which the HERA experiments are more sensitive than experiments at LEP and the TeVatron.

\section{References}

[1] H1 Coll., V. Andreev et al., Phys. Lett. B 561 (2003) 241;

[2] K.-P. O. Diener, C. Schwanenberger and M. Spira, Eur. Phys. J. C 25 (2002) 405.

[3] H1 Coll. A. Aktas et al., EPS2005 contr. paper, abstr. 637;

[4] ZEUS Coll. S. Chekanov et al., EPS2005 contr. paper, abstr. 327;

\footnotetext{
${ }^{1)}$ Where V denotes a neutral vector boson.
} 


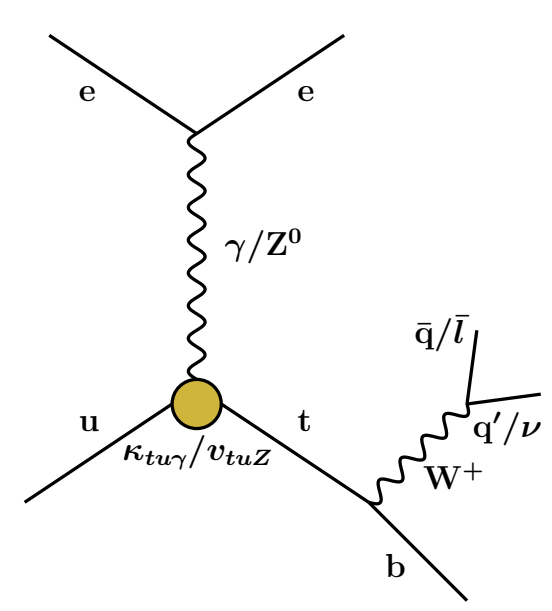

(a)

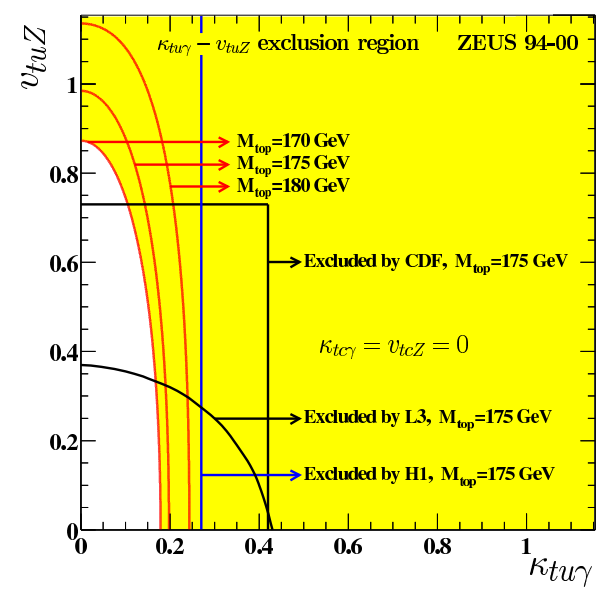

(b)

Figure 2: (a) Feynman diagram for single-top prodcution via FCNC transitions at HERA (b) The existing limits on FCNC from the TeVatron, LEP and HERA.

[5] ZEUS Coll. S. Chekanov et al., Phys. Lett. B 583 (2004) 41;

[6] H1 Coll. A. Aktas et al., EPS2005 contr. paper, abstr. 624;

[7] S.L. Glashow, J. Iliopoulos and L. Maiani, Phys. Rev. D 2 (1970) 1285.

[8] H. Fritzsch and D. Holtmannspötter, Phys. Lett. B 457 (1999) 186.

[9] A. Belyaev and N. Kidonakis, Phys. Rev. D 65 (2002) 037501.

[10] ZEUS Coll., S. Chekanov et al., Phys. Lett. B 559 (2003) 153;

[11] H1 Coll., A. Aktas et al., Eur. Phys. J. C33 (2004) 9 ,

[12] CDF Coll., F. Abe et al., Phys. Rev. Lett. 80 (1998) 2525.

[13] L3 Coll., P. Achard et al., Phys. Lett. B 549 (2002) 290. 